\title{
DIVERSIDAD DE FLORA NATIVA POR ESCALAS DE ALTITUD EN LA AMAZONÍA FRIA
}

\section{ESTUDIO PILOTO EN LA ESTACIÓN BIOLÓGICA PINDO MIRADOR}

\author{
Autora: \\ Carmen Luzuriaga'
}





\section{Resumen}

El presente estudio se realizó en la Estación Biológica Pindo Mirador con el objetivo de identificar las especies de flora en diferentes áreas de acuerdo a la gradiente altitudinal. Los bosques de la Estación Biológica Pindo Mirador son ecosistemas de la Amazonía donde habitan una variedad de especies; se trabajó con transectos radiales de tal forma que esta metodología permitió cubrir un mayor número de especies.

Los datos obtenidos fueron analizados y se estableció la composición florística, índice de diversidad, índice de similitud y el estado de conservación de las especies de bosque primario y secundario. La densidad fue mayor en los bosques secundarios, mientras la diversidad de acuerdo al índice obtenido es medianamente baja, siendo la similitud entre los transectos establecidos más similar a los 1300 metros. Los bosques secundarios se encuentran en un estado de recuperación.

\section{Abstract}

This study was conducted at the Biological Station Pindo Mirador in order to identify the species of flora in different areas according to the altitudinal gradient. The forests of the Biological Station Pindo Mirador are ecosystems of the Amazon inhabited by a variety of species we worked with radial transects so that this methodology allowed to cover a larger number of species.

The data were analyzed and determined the composition of floristic diversity index, similarity index and the conservation status of species of primary and secondary forest. The density was higher in secondary forests, while according to the diversity index obtained is fairly low, with the similarity between the transects established more similar to 1300 meters. Secondary forests are in a state of recovery.

Palabras clave: Especies florísticas, bosque piemontano, diversidad, similitud, altitud, endémico.

Recibido: Mayo 2011

Aprobado: Julio 2011 


\section{Introducción}

El estudio realizado en los bosques de la Estación Biológica Pindo Mirador, ubicados en el cantón Mera, provincia de Pastaza, destaca la importancia de los recursos que guardan en los ecosistemas y la relación interespecífica que comparten las especies en la naturaleza.

Este estudio se realizó a lo largo de la gradiente altitudinal con el objetivo de identificar las especies de flora y qué cambios ocurrían en la vegetación a manera que se ascendía; por ello la altitud fue una variable que se consideró importante y bajo la cual se establecieron los transectos de investigación en 0,6 hectáreas de bosque primario y secundario.

El muestreo realizado permitió establecer la composición florística, índice de diversidad, índice de similitud y el estado de conservación de las especies de bosque primario y secundario bajo un análisis riguroso de los datos obtenidos.

Los resultados demuestran que los bosques de la Estación Biológica presentan a mayor altitud mayor número de especies; lo cual en la discusión se plantea que los procesos de colonización, la intervención antropogénica en el bosque produjo la explotación de este recurso en las partes bajas donde se realizó el estudio. Las condiciones ambientales influyen decididamente en la tolerancia de algunas especies en el tiempo y el espacio lo cual determina la densidad y diversidad de éstas en los diferentes rangos altitudinales. La densidad del bosque secundario es mayor en relación con el bosque primario. Este dato permite establecer que el crecimiento de varias especies es simultáneo en bosque secundario, el cual se evidencia al haber sido registradas 16 familias con una sola especie, lo que determina que el bosque está en una etapa de recuperación. De allí que existan individuos jóvenes y compitan entre sí por asimilar mejor los nutrientes, el agua y la energía de tal forma que a futuro tendrán un mayor valor de importancia en el bosque.
Pese a que la diversidad registrada se interpreta como medianamente baja la recuperación del bosque secundario es óptima de tal forma que a futuro estos bosques servirán de material genético y germoplasma para las futuras generaciones.

La sucesión "es el cambio progresivo de la comunidad a través del tiempo en respuesta al cambio de las condiciones ambientales"'; esta característica ambiental permitirá a los bosques secundarios alcanzar un mejor estadio hasta convertirse en bosques prístinos y asegurar de esta forma la dinámica y cadenas tróficas en la naturaleza.

\section{Objetivos}

\section{Objetivo General}

- Identificar las especies de flora en diferentes áreas de acuerdo a escalas de altitud en el bosque piemontano de la Estación Biológica Pindo-Mirador.

\section{Objetivos Específicos}

- Inventariar las especies de flora por escalas de altitud - Determinar el valor de importancia de las especies florísticas. - Determinar la diversidad en las diferentes áreas de estudio.

- Establecer la similitud que presentan las especies influenciadas gradientes altitudinales.

- Establecer un análisis cuantitativo de las especies florísticas. 


\section{Metodología}

La metodología empleada requirió dividir en dos fases el trabajo:

\section{Fase de campo}

De acuerdo a las salidas exploratorias y de observación se establecieron en los bosques de la Estación transectos radiales, los cuales permitieron registrar especies florísticas con una composición muy heterogénea. De acuerdo a la metodología empleada por el Botánico Carlos Cerón (2005), para bosque húmedo premontano recomienda establecer 5 transectos de $50 \times 4$ metros. Para el presente estudio se trazaron seis transectos; los cuales fueron distribuidos tres en bosque primario y tres en bosque secundario.

\section{BOSQUE SECUNDARIO}

\begin{tabular}{|c|c|c|c|}
\hline & \multicolumn{3}{|c|}{ ALTITUD } \\
\hline TRANSECTO & 1193 & 1250 & 1300 \\
\hline A & $\mathrm{X}$ & & \\
\hline B & & $\mathrm{X}$ & \\
\hline C & & & $\mathrm{X}$ \\
\hline
\end{tabular}

En Bosque Secundario se ubicaron los transectos de acuerdo a la gradiente altitudinal cada uno con 50 metros aproximadamente de diferencia. La altitud se ha medido utilizando un altímetro SUUNTO con el cual se ha comprobado la ubicación de los transectos.

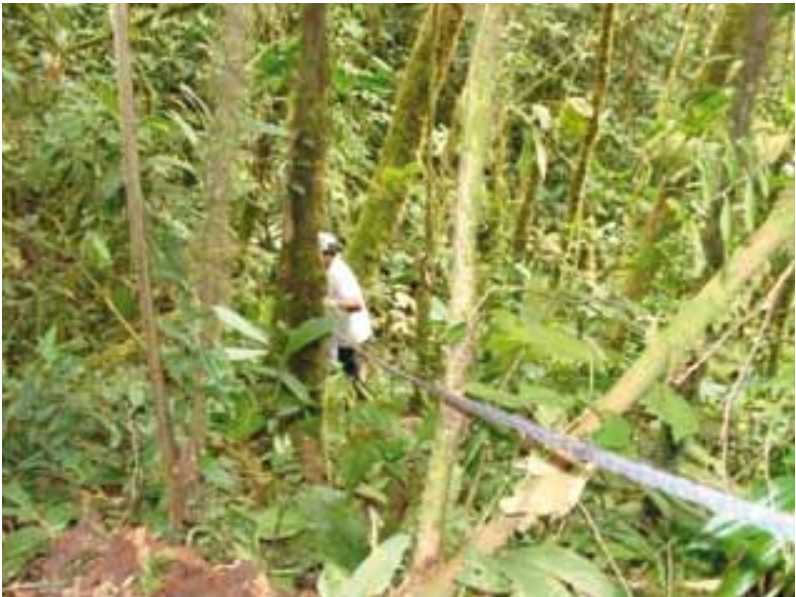

Foto 1. Trazando transectos en bosques de la EBPM.
El transecto A se ubica a 1193 metros, el transecto B a 1250 metros y el transecto $\mathrm{C}$ a 1300 metros.

\section{BOSQUE PRIMARIO}

\begin{tabular}{|c|c|c|c|}
\hline & \multicolumn{3}{|c|}{ ALTITUD } \\
\hline TRANSECTO & 1280 & 1290 & 1300 \\
\hline D & X & & \\
\hline E & & $\mathrm{X}$ & \\
\hline F & & & $\mathrm{X}$ \\
\hline
\end{tabular}

La Estación Biológica Pindo Mirador tiene bosque primario, que de acuerdo con el altímetro los sitios escogidos, no se encuentran a altitudes bajo los 1280 metros y los sitios más altos llegan hasta los 1300 metros. Por esta situación se consideró un rango de 10 metros de altitud entre los diferentes transectos. El transecto D se ubica a 1280 metros, el transecto E se ubica a 1290 metros y el transecto C a 1300 metros.

\section{Transectos de Investigación}

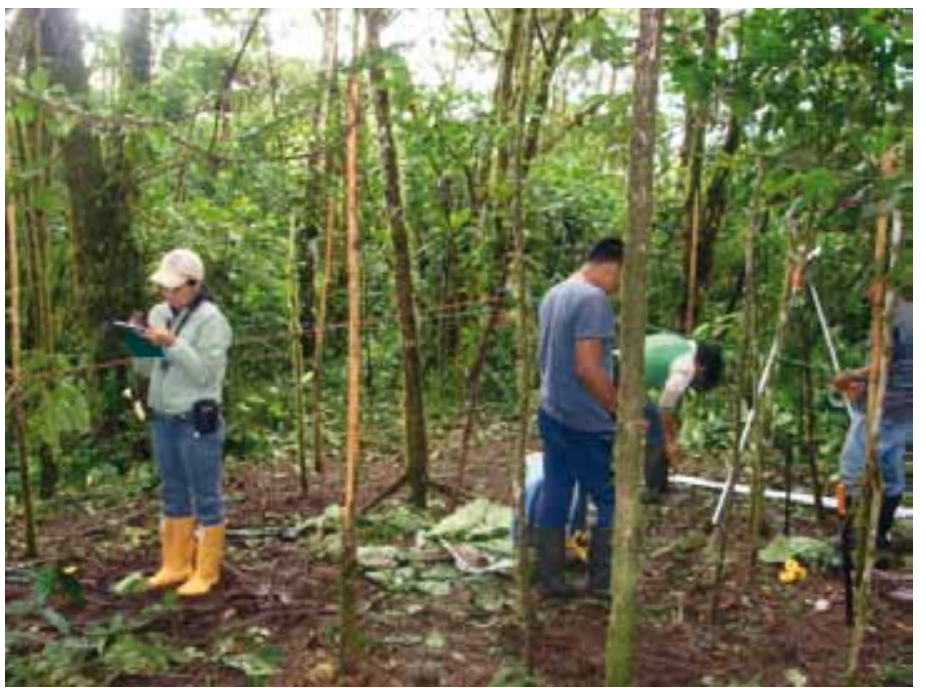

Foto 2. Transectos radiales en Bosque Secundario

Los transectos son espacios delimitados de vegetación.

Tamaño: Cada transecto radial tiene $50 \times 4$ metros, que 
representa 0,1 $\mathrm{Ha}$. Las especies analizadas fueron $\geq 2,5 \mathrm{~cm}$ de DAP

(Diámetro a la altura del pecho).

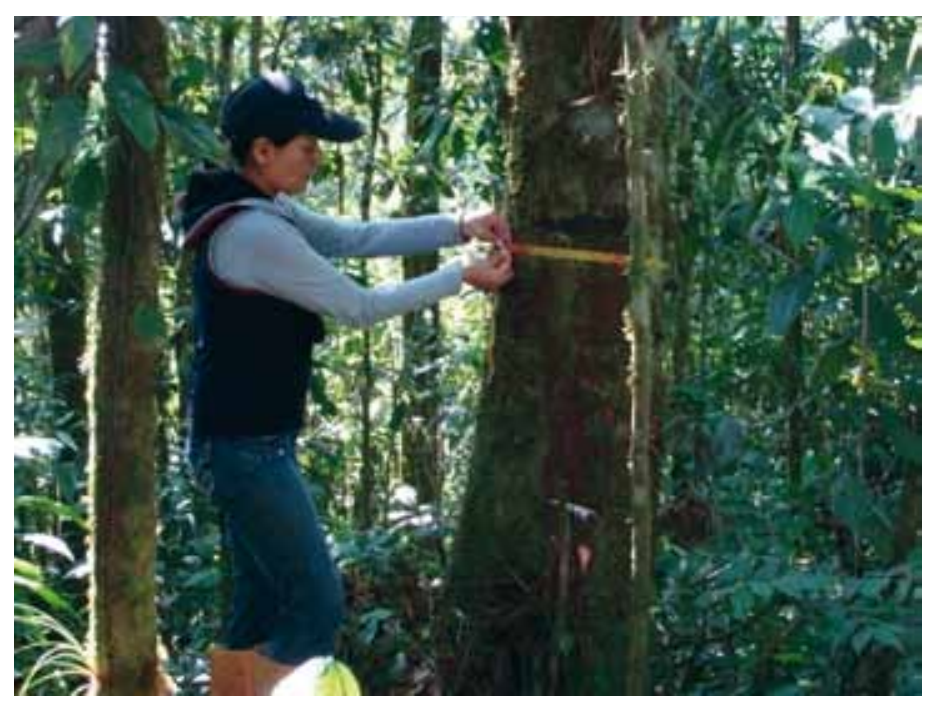

Foto 3. Medición del DAP

Las especies fueron registradas en la libreta de campo además se fue colectando las muestras con su respectiva codificación.

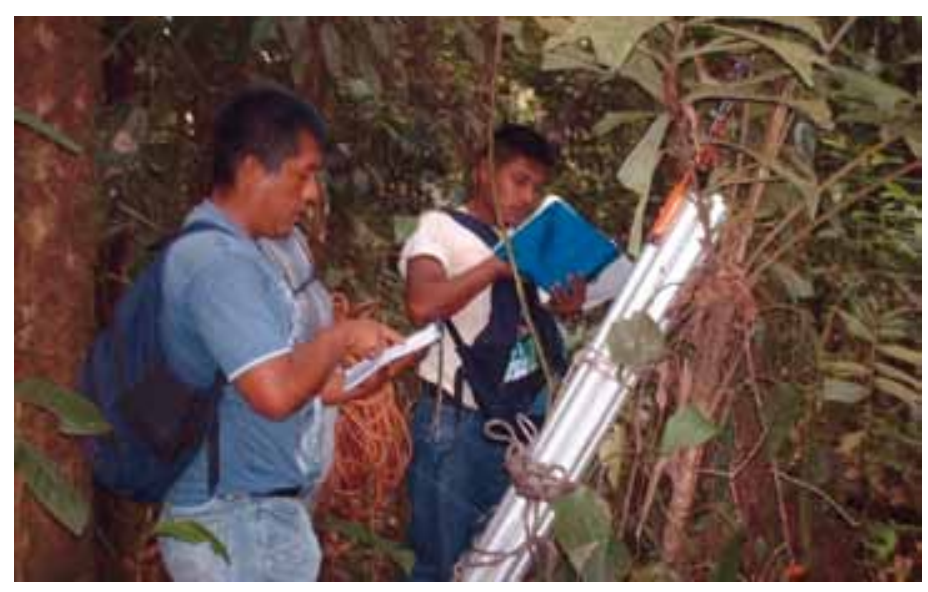

Foto 4. Revisando datos en libreta de campo.

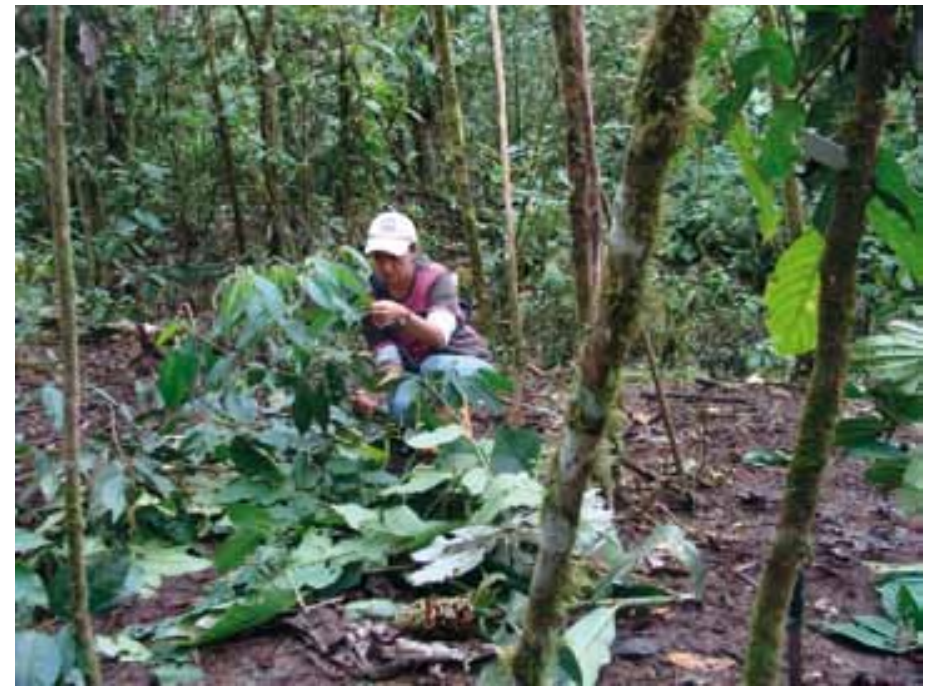

Foto 5. Muestras de árboles para prensar

Fase de Laboratorio

Las muestras colectadas en el bosque fueron ordenadas y clasificadas para prensarlas. La etapa de prensado se realizó en periódico procurando no dañar las muestras botánicas, para lo cual es necesario utilizar como preservante alcohol potable.

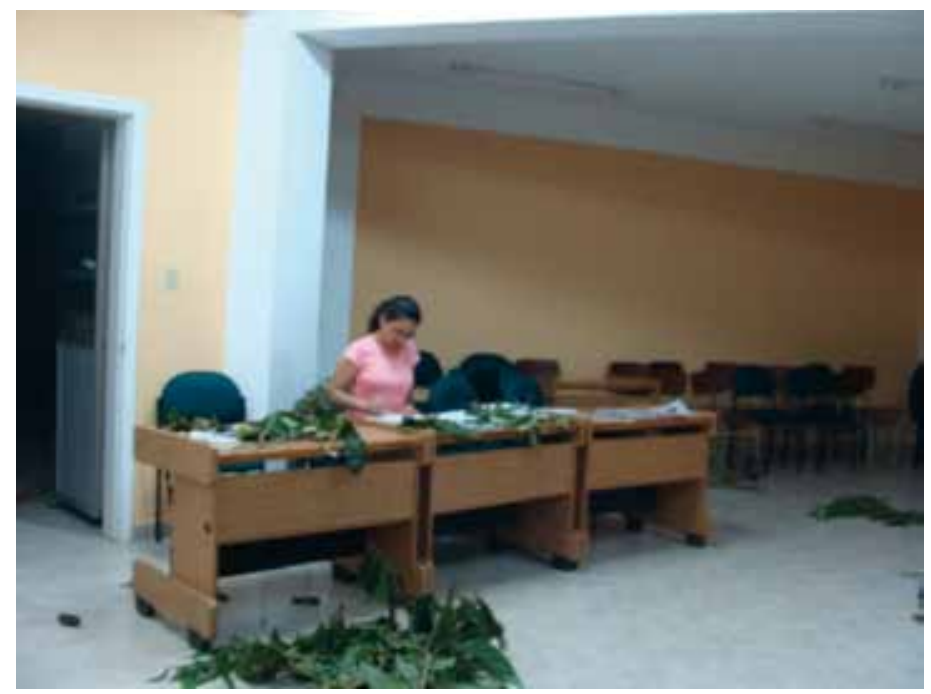

Foto 6. Prensando muestras botánicas en el Laboratorio.

Las muestras prensadas fueron enviadas a Quito al Herbario Nacional del Ecuador (QCNE) donde se realizó el secado e identificación.

68

TsaFiquili:msand 


\section{Análisis de datos.}

Indice de Diversidad de Simpson de acuerdo a Cerón (2003) y Krebs (1985).

Cuantifica la diversidad de especies florísticas.

$$
\mathrm{IDS}=\frac{1}{\sum \mathrm{Pi}^{2}}
$$

Donde:

IDS = Índice de Diversidad de Simpson corregido

$\Sigma=$ Sumatoria

1 = Constante del Índice de Simpson corregido

$\mathrm{Pi}^{2}=$ Proporción de individuos elevado al cuadrado

Índice de Valor de Importancia, para cada una de las especies. (Campbell 1989).

\section{Fórmulas para el cálculo del I.V.I}

Área Basal (AB)

$$
\mathrm{AB}=\frac{\pi \mathrm{D}^{2}}{4}
$$

Donde:

$\mathrm{D}=$ Diámetro

Índice de Valor de importancia (I.V.I.)

$$
I . V . I=D n R+D m R .
$$

Donde:

Dn. $R=$ Densidad Relativa

$=$ (No. de árboles de la especie / No. total de las especies)

$\mathrm{x} 100$

Dm. $R=$ Dominancia Relativa

= (Área Basal de la especie / Área basal total de las especies)

x100

\section{Indice de Similitud}

Indice de Similitud de Sorensen

$I S S=2 C / A+B$

ISS=Índice de Similitud de Sorensen

$A=$ Número de especies del muestreo $A$

$\mathrm{B}=$ Número de especies del muestreo $\mathrm{B}$

$C=$ Número de especies compartidas entre el muestreo $A$ y $B$

Indice de Diversidad de Simpson de acuerdo a Cerón (2003) y Krebs (1985).

Cuantifica la diversidad de especies florísticas.

$$
\mathrm{IDS}=\frac{1}{\sum \mathrm{Pi}^{2}}
$$

Donde:

IDS = Índice de Diversidad de Simpson corregido

$\Sigma=$ Sumatoria

1 = Constante del Índice de Simpson corregido

$\mathrm{Pi}^{2}=$ Proporción de individuos elevado al cuadrado

Índice de Valor de Importancia, para cada una de las especies. (Campbell 1989).

\section{Fórmulas para el cálculo del I.V.I}

Área Basal (AB)

$$
\mathrm{AB}=\frac{\pi \mathrm{D}^{2}}{4}
$$

Donde:

$\mathrm{D}=$ Diámetro 
Índice de Valor de importancia (I.V.I.)

$$
\text { I.V.I }=\text { DnR + DmR. }
$$

Donde:

Dn. $\mathrm{R}=$ Densidad Relativa

= (No. de árboles de la especie / No. total de las

especies)x100

Dm.R = Dominancia Relativa

= (Área Basal de la especie / Área basal total de las especies) $\times 100$

\section{Indice de Similitud}

Indice de Similitud de Sorensen

$\mathrm{ISS}=2 \mathrm{C} / \mathrm{A}+\mathrm{B}$

ISS=Índice de Similitud de Sorensen

$A=$ Número de especies del muestreo $A$

$B=$ Número de especies del muestreo $B$

$\mathrm{C}=$ Número de especies compartidas entre el muestreo $\mathrm{A}$ y $\mathrm{B}$

\section{Determinación del Endemismo}

El endemismo se determinó utilizando el Catálogo de Plantas Vasculares del Ecuador (Jogersen y León 1999).

\section{Materiales}

- 2 podadoras aéreas

- 2 podadoras manuales

- 5 galones de alcohol

- 1 cámara digital

-1 GPS

- 2 brújulas

- 1 altímetro

-5 rollos de soga

\author{
-5 rollos de piola \\ - 2 libretas de campo \\ - cinta diamétrica \\ - maskin \\ - fundas plásticas \\ - fundas de papel \\ - trepadoras \\ - esferos \\ - papel periódico \\ - marcadores indelebles \\ - papel bond \\ - pintura \\ - placas metálicas
}

\section{Resultados}

\section{Análisis cuantitativo. Estructura y Composición Florística.}

\section{Bosque Primario}

En el muestreo realizado utilizando la metodología de transectos radiales en Bosque Primario de la E.B.P.M. en 0,3 hectáreas de esta investigación se registraron 510 individuos de más de $2,5 \mathrm{~cm}$ de DAP, que corresponden a 105 especies, 71 géneros y 38 familias botánicas.

Las familias con mayor número de especies son: Rubiaceae (13), Lauraceae (9), Melastomataceae (9), Mimosaceae (7), Euphorbiaceae (7) Meliaceae (7); mientras que familias como Arecaceae tienen 4 especies y familias con 3 especies se registran las siguientes: Annonaceae, Bombacaceae, Clusiaceae, Moraceae, Myrtaceae y con 2 especies respectivamente se registran Cecropiaceae, Flacuortiaceae, Icacinaceae, Monimiaceae, Myristicaceae, Nyctaginaceae, Rutaceae y familias con una especie: Actinidaceae, Apocynaceae, Araliaceae, Asteraceae, Burseraceae, Caesalpinaceae, Caprifoliaceae, Cunoniaceae, Cyatheaceae, 
Elaeocarpaceae, Ericaceae, Lecythidaceae, Myrsinaceae, Piperaceae, Poaceae, Protaceae, Solanaceae, Staphylaceae y Violaceae.

\section{Bosque Secundario}

En el muestreo realizado utilizando la metodología de transectos radiales en Bosque Secundario de la E.B.P.M. en 0,3 hectáreas de esta investigación se registraron 711 individuos de más de 2,5 cm de DAP, que corresponden a 101 especies, 62 géneros y 35 familias botánicas.

Las familias con mayor número de especies son: Rubiaceae (12), Melastomataceae (11), Mimosaceae (10), Euphorbiaceae (7), Lauraceae (7), Myrtaceae (6); mientras que, familias como Arecaceae, Cecropiaceae, Meliaceae tienen 4 especies, Rutaceae con 3 especies y Annonaceae, Caprifoliaceae, Clusiaceae, Cyatheaceae, Monimiaceae, Myrsinaceae, Piperaceae, Solanaceae, y Vochysiaceae con 2 especies respectivamente; y con una especie se registran Actinidaceae, Anacardiaceae, Asteraceae, Bombacaceae, Brunelliaceae, Burseraceae, Caesalpinaceae, Costaceae, Elaecarpaceae, Flacuortaceae, Lacistamataceae, Moraceae, Protaceae, Staphylaceae, Sterculiaceae y Urticaceae.

\section{Especies importantes para la conservación y especies}

\section{endémicas}

Los bosques de la Amazonía de acuerdo al Libro Rojo de Plantas Vasculares presentan bajos niveles de endemismo, “las plantas endémicas del Ecuador están abrumadoramente concentradas en la zona andina, con una menor proporción en las tierras bajas de la Costa y una cantidad pequeña restringida a las tierras bajas de las Galápagos y la Amazonía."

Las especies registradas como endémicas en este estudio son las siguientes: Coussarea ecuadorensis (Burseraceae), Inga multinervis (Fab-Mimosaceae), Miconia cercophora (Melastomataceae), Miconia

2 Valencia, R. (2000). Libro Rojo de las Plantas Endémicas del Ecuador. Pág. 17 lugonis (Melastomataceae), Saurauia aequatoriensis (Actinidaceae). Saurauia aequatoriensis está catalogada en la categoría de preocupación menor (LC), así como las dos especies Miconia cercophora y Miconia lugonis en la categoría de Casi Amenazado (NT) de acuerdo a la UICN.

\section{Códigos UICN:}

EX $=$ Extinta

$\mathrm{EW}=$ Extinta en la Naturaleza

$\mathrm{CR}=$ En Peligro Crítico

$\mathrm{EN}=$ En Peligro

$\mathrm{VU}=$ Vulnerable

NT = Casi Amenazada

LC = Preocupación Menor

DD = Datos Insuficientes

$\mathrm{NE}=$ No Evaluada

Símbolos: $†$ = Se conoce únicamente el tipo

* = No confirmada dentro del SNAP.

Las categorías de amenaza se aplicaron de acuerdo a la categorización de la Unión Internacional para la Conservación de la Naturaleza (UICN). Para la revisión de cada una de las especies se utilizó a Valencia, R., Pitman, N., S. León-Yánez \& P. M. Jorgensen (eds.) (2000).

\section{Discusión}

Los bosques de la Estación Biológica Pindo Mirador se agrupan en dos tipos: Bosque Primario y Bosque Secundario.

Las Familias botánicas más representativas para bosque primario y secundario son: Rubiaceae, Melastomataceae, Lauraceae, Euphorbiaceae, Mimosaceae pero principalmente la Rubiaceae; la cual está ampliamente distribuida en las diferentes formaciones boscosas del país y específicamente en todas las gradientes altitudinales 
identificadas en este estudio desde los 1193m hasta los 1300 metros a los que encuentran los bosques piemontanos de la Estación Biológica Pindo Mirador.

El Bosque Secundario es un ecosistema donde el dosel oscila entre los 15 y 20 metros, el sotobosque es denso, se encuentran gran cantidad de arbolitos en crecimiento, hierbas gigantes; la elevada densidad puede estar dada por la presencia de individuos jóvenes y pocos árboles de gran tamaño lo cual se pudo evidenciar al registrar 16 familias botánicas con una sola especie. En Bosque Secundario a diferentes altitudes a las que se ubicaron los transectos la densidad de sus individuos fue mayor que en relación a los transectos en Bosque Primario; de tal forma que, la densidad a lo largo del gradiente altitudinal refleja cómo el medio actúa sobre las especies florísticas.

En cuanto a la composición florística en el bosque secundario se registró en 0,3ha muestreadas 101 especies, la mayor cantidad (61) se encontró a los 1250 metros; mientras que, en los 1193 metros se registraron menos especies (34). A pesar de que la diversidad y la composición florística fueron diferentes a lo largo de la gradiente altitudinal se puede establecer que las condiciones de recuperación del bosque secundario aseguran su permanencia y desarrollo a un mejor estadío.

El valor de importancia de las especies florísticas entre las que se destacan Miconia splendens, Piptocoma discolor, Cecropia angustifolia, Saurauia aequatoriensis, Palicourea gomezii, Cecropia ficifolia cuyo interés implica que al absorber gran cantidad de nutrientes conlleva a que ocupen mayor espacio físico en el bosque y controlen un elevado porcentaje de energía que llega al sistema.

El Bosque secundario se registró a partir de los 1193m hasta los 1300m; en el estudio realizado a los 1250 metros disminuyó la densidad de los árboles y aumentó el número de especies. Parece ser que a esta altitud la recuperación de este ecosistema boscoso mejoró; lo cual puede deberse a que los cambios sucesionales de bosque se han producido de mejor manera a esta altitud a lo largo de la gradiente. Al mismo tiempo se registró que a 1193 metros se encontraron 34 especies y a 1300 metros 47 especies; lo cual puede interpretarse que a mayor altitud hay mayor cantidad de especies.

La intervención antropogénica que sufrieron estos bosques jugó un papel preponderante y fue quizás la causa directa que afectó la diversidad del bosque, es decir que no ha sido por procesos naturales ni ecológicos que ha ocurrido esta situación; pues mientras más bajo se encontraban los bosques fueron más fáciles de explotarlos; de allí que a mayor altitud se hayan conservado de mejor manera.

El Bosque Primario se presenta como un ecosistema donde el dosel oscila entre los 20 y 30 metros, el sotobosque es menos denso, la presencia de árboles maduros y gruesos como una característica de este potencial ecológico registrado desde los $1280 \mathrm{~m}$ hasta los 1300 metros. La altitud y la distancia a la que se registran este tipo de bosque han permitido la ausencia del hombre por lo que su estado es estable. De allí que se registraron 105 especies de 510 individuos muestreados siendo las especies más frecuentes Wettinia maynensis, Caliandra trinervia, Palicourea hospitalis, Palicourea acanthacea, Viburnum ayavecense, Zygia coccínea y Miconia trinervia.

En cuanto a la composición florística en el bosque primario se registró en 0,3ha muestreadas 105 especies, la mayor cantidad (59) se encontró a los 1300 metros; mientras que, en los 1280 metros se registraron (57). La diversidad y la composición florística se mantienen en valores casi permanentes, de tal forma que la diferencia establecida en los transectos que se trabajaron con un rango cada 10 metros en realidad no fue para este estudio significativo ya que se evidenciaron en los resultados que a 1280 y 1300 metros la diversidad y la composición no varían significativamente, lo cual si varió a los 1290 metros pues disminuyó el número de especies y la densidad del bosque. Quizás la explicación más razonable que se puede plantear es que a esta altitud el bosque se encuentra en un una franja más bien de transición o estado de transición que puede ser 
parte de cualquiera de los otros dos bosques ya sea a mayor o menor altitud. En el Bosque Primario se evidenciaron que a 1280 metros se registraron 57 especies mientras a los 1300 metros 59 especies; lo cual puede interpretarse que el número de especies o diversidad alfa es constante y se mantiene con la altitud puesto que están en una misma franja de vegetación.

La mayor diversidad de especies (59) se registró a más altitud de los 1300 metros, lo cual puede ser debido a que en estas zonas la topografía del suelo no es tan irregular como sucedió en los otros transectos; lo que ha permitido un mejor desarrollo de las especies.

El valor de importancia de las especies florísticas entre las que se destacan : Wettinia maynensis, Alchornea latifolia, Zygia coccínea, Pouruma cecropifolia, Protium amazonicum, Calliandra trinervia, Palicourea hospitalis, Viburnum ayavecense, Inga tenuistipula, Palicourea acanthacea cuyo interés implica que al ser grandes árboles llegan a absorber mayor cantidad de nutrientes por lo cual la densidad es menor en este bosque; siendo especies dominantes ecológicamente ante los otros individuos que comparten este hábitat.

El índice de Diversidad en los diferentes transectos establecidos a lo largo de la gradiente altitudinal evidenció que tanto para Bosque Secundario y Primario se interpreta como una diversidad medianamente baja, lo cual puede deberse a que el bosque secundario fue explotado y en el bosque primario las frecuentes lluvias y árboles que caen por las tormentas destruyen especies que no son tan resistentes y predominan aquellas que tienen altas densidades.

El índice de similitud de los transectos de Bosque Primario y Secundario es del $42 \%$, lo cual significa que la composición florística entre los dos bosques es bastante similar y a la vez es un indicador de que el bosque secundario se encuentra en un estado óptimo de recuperación.

Los Bosques de la Estación Biológica Pindo Mirador representan un gran potencial ecológico, natural, ambiental y el estudio establecido revela de forma interesante que pese a que no se registró gran diversidad los bosques se encuentran en un estado de óptima conservación y en el futuro se convertirán en fuentes potenciales de germoplasma y bancos genéticos de la Alta Amazonía.

\section{Conclusiones}

El bosque primario mantiene una densidad menor de individuos en relación al bosque secundario. Los grandes y gruesos árboles asimilan gran cantidad de nutrientes y mantienen una estrecha relación con el ambiente por lo que se registraron 105 especies distribuidos en la gradiente altitudinal de la siguiente forma: a los 1280 metros 56 especies, a los 1290 metros 42 especies y a los 1300 metros 59 especies.

El bosque secundario presenta mayor densidad siendo característico la presencia de árboles jóvenes y delgados distribuidos en la gradiente altitudinal de la siguiente forma: a los 1193 metros 34 especies, a los 1290 metros 61 especies y a los 1300 metros 47 especies.

La composición florística en bosque primario está dada por las siguientes familias Rubiaceae, Lauraceae, Melastomataceae, Mimosaceae, Euphorbiaceae y Meliaceae; mientras en bosque secundario se registran las anteriormente mencionadas y una más, la Myrtaceae.

En el muestreo realizado utilizando la metodología de transectos radiales en Bosque Primario de la E.B.P.M. en 0,3 hectáreas se registraron 510 individuos de más de 2,5cm de DAP, que corresponden a 105 especies, 71 géneros y 38 familias botánicas.

En el muestreo realizado utilizando la metodología de transectos radiales en Bosque Secundario de la E.B.P.M. en 0,3 hectáreas se registraron 711 individuos de más de 2,5 cm de DAP, que corresponden a 101 especies, 62 géneros y 35 familias botánicas. 
Deacuerdo al muestreo realizado el género más representativo tanto para bosque primario y secundario es Miconia de la familia Melastomataceae con 8 y 11 especies respectivamente.

En bosque primario en los transectos establecidos de acuerdo al valor de importancia se destacan las siguientes especies: a los 1280 Ocotea sp. $(38,96)$, a los 1290 Protium amazonicum $(34,41)$ y a los 1300 metros Alchornea latifolia $(22,91)$.

En bosque secundario en los transectos establecidos de acuerdo al valor de importancia se destacan las siguientes especies: a los 1193 Miconia splendens (37,62), a los 1250 Lozania klugii $(40,20)$ y a los 1300 metros Miconia trinervia $(73,37)$.

El índice de diversidad para bosque secundario en los distintos transectos se interpreta como una diversidad beta de los muestreos realizados a los 1300 metros es la más alta con 91 especies a diferencia de los otros muestreos realizados a menor altitud.

Es interesante destacar que de acuerdo al índice de similitud a 1300 metros entre los transectos establecidos en bosque primario y bosque secundario el porcentaje de similitud alcanza el $34 \%$ por lo que presentan similitud sus especies.

La recuperación del bosque es óptima. Existe una competencia de las especies dentro de un equilibrio natural lo cual determina el cambio a un nuevo estadio.

La ausencia del hombre en actividades de explotación y destrucción del bosque ha determinado la recuperación y conservación del bosque, mientras que, la presencia de animales dispersores y polinizadores de semillas garantiza su recuperación.

Las especies registradas como endémicas en este estudio son las siguientes: Coussarea ecuadorensis (Burseraceae), Inga multinervis (Fab-Mimosaceae), Miconia cercophora (Melastomataceae), Miconia lugonis (Melastomataceae), Saurauia aequatoriensis (Actinidaceae), todas sin peligro de extinción.

\section{Recomendaciones}

- Mantener los estudios florísticos ya que ayudan a conocer la dinámica del bosque y el comportamiento que tienen las especies en el tiempo y en el espacio.

-Mantener lineamientos que protejan los bosques de la Estación Biológica Pindo Mirador ya que son lugares que se encuentran en proceso de recuperación y otros sirven de bancos de semillas. 


\section{Bibliografía}

Cañadas, L. (1983). Mapa bioclimático y ecológico del Ecuador. MagPronareg y Banco Central del Ecuador, Quito.

Cerón, C.E. (1993). Impactos sobre la vegetación en áreas naturales del Ecuador. Geográfica (Quito) 32: 99-118.

Cerón, C.E. (2003). Manual de botánica, sistemática, etnobotánica y métodos de estudio en el Ecuador. Herbario Alfredo Paredes (QAP, Escuela de Biología de la Universidad Central del Ecuador, Editorial Universitaria, Quito.

Font Quer, Pío (1980). Plantas Medicinales. Editorial Labor S.A., Barcelona.

Freire Fierro, Alina (2004). Botánica Sistemática Ecuatoriana. Fundacyt. Quito.

Jiggins, C., Andrade, P., Cueva, E., Dixon S., Isherwood I. \& Willis J. (2000). Flora y Fauna. Guía del Sur Occidente del Ecuador, Lone Pine Plblishing, Cánada.

Jørgensen, P.M.y S. León-Yánez (eds.). (1999). Catalogue of the vascular plants of Ecuador. Missouri Botanical Garden Press, St. Louis, Missouri.

Jolitz, T., Kleinn C. \& Palacios W. (2001). Manual para estudios científicos en forestería, agricultura y ecología. Fundación Jatun Sacha. Quito- Ecuador.

Luzuriaga, C. (2007). Diagnóstico de la Flora Estación Biológica Pindo Mirador. CODEU. Quito- Ecuador.

Morocho, D. \& C. Romero. (Eds.) 2003. Bosques del Sur. El estado de 12 remanentes de bosques andinos de la provincia de Loja. Fundación Ecológica Arcoiris. Probona/Dica. Loja- Ecuador.

Ríos, M., Koizol J., Borgtoft Pedersen H. \& Granda G. (Eds.) (2007). Plantas Útiles del Ecuador: aplicaciones, retos y perspectivas. Ediciones Abya -Yala, Quito- Ecuador.
Vásquez, Rodolfo \& Rojas Rocío del Pilar (2003). Plantas de la Amazonía Peruana. Missouri Botanical Garden, Perú.

Villarroel, Francisco (1991). Introducción a la Botánica Sistemática. Editorial Universitaria, Quito-Ecuador. 


\section{ANEXOS}

Cuadro1. Especies registradas en Bosque Primario

\begin{tabular}{|c|c|c|c|c|}
\hline & FAMILIA & ESPECIE & NOMBRE COMÚN & $\mathrm{F}$ \\
\hline 1 & Actinidaceae & Saurauia aequatoriensis & Moquillo & 2 \\
\hline 2 & Annonaceae & Annona duckei & Anona demonte & 1 \\
\hline 3 & Annonaceae & Guatteria sp. & Caracaspi & 2 \\
\hline 4 & Annonaceae & Guatteria sp. 1 & Caracaspi & 1 \\
\hline 5 & Apocynaceae & Tabernaemontana amygdalifolia & Pata de danta & 1 \\
\hline 6 & Araliaceae & Oreopanax palamophyllus & Pata de danta & 2 \\
\hline 7 & Arecaceae & Prestoea acuminata & Chontilla & 4 \\
\hline 8 & Arecaceae & Wettinia maynensis & Quilo & 44 \\
\hline 9 & Arecaceae & Prestoea schultzeana & Chontilla con espina & 2 \\
\hline 10 & Arecaceae & Geonoma interrupta & Ucsha & 2 \\
\hline 11 & Asteraceae & Piptocoma discolor & Pigue & 1 \\
\hline 12 & Bombacaceae & Matisia lasiocalyx & Sapote macho & 4 \\
\hline 13 & Bombacaceae & Matisia soegengii & Sapote & 2 \\
\hline 14 & Bombacaceae & Matisia ochrocalyx & Sapota macho & 1 \\
\hline 15 & Burseraceae & Protium amazonicum & Copal & 7 \\
\hline 16 & Caesalpinaceae & Macrolobium acaciiflolium & Guarango & 1 \\
\hline 17 & Caprifoliaceae & Viburnum ayavacense & Yacu caspi blanco & 18 \\
\hline 18 & Cecropiaceae & Cecropia angustifolia & Guarumo & 5 \\
\hline 19 & Cecropiaceae & Pourouma cecropiifolia & Uva & 13 \\
\hline 20 & Clusiaceae & Tovomita wedelliana & Yacu caspi de pantano & 13 \\
\hline 21 & Clusiaceae & Chrysochlamys membranaceae & Manzano & 6 \\
\hline 22 & Clusiaceae & Vismia pozuzoensis & Achotillo BP & 1 \\
\hline 23 & Cunoniaceae & Weinmannia lentiscifolia & Arrayan traposo & 3 \\
\hline 24 & Cyatheaceae & Cyathea sp. & Helecho & 8 \\
\hline 25 & Elaeocarpaceae & Sloanea guianensis & Manduro caspi & 2 \\
\hline 26 & Ericaceae & Cavendishia tarapotana & Gualicón & 1 \\
\hline 27 & Euphorbiaceae & Pausandra sp. & Caluncalun & 2 \\
\hline 28 & Euphorbiaceae & Alchornea latifolia & Ponce & 22 \\
\hline 29 & Euphorbiaceae & Sapium marmieri & Caucho & 13 \\
\hline 30 & Euphorbiaceae & Croton tessmannii & Agujero & 3 \\
\hline 31 & Euphorbiaceae & Croton lechleri & Drago & 1 \\
\hline 32 & Euphorbiaceae & Sapium peruvianum & Caucho & 2 \\
\hline 33 & Euphorbiaceae & Hyeronima scabrida & Motilón blanco & 7 \\
\hline 34 & FAB-MIM & Inga velutina & Guabilla & 4 \\
\hline
\end{tabular}




\begin{tabular}{|c|c|c|c|c|}
\hline 35 & FAB-MIM & Inga tenuistipula & Guabilla & 10 \\
\hline 36 & FAB-MIMO & Inga multinervis & Guaba de rio & 2 \\
\hline 37 & FAB-MIMO & Inga stipulacea & Guaba & 4 \\
\hline 38 & FAB-MIMO & Ormosia sp. & Rayu caspi & 1 \\
\hline 39 & FAB-MIMOS & Zygia coccinea & Guabilla & 16 \\
\hline 40 & Fab-Mimosaceae & Calliandra trinervia & Yutzu & 22 \\
\hline 41 & Flacourtiaceae & Casearia sylvestris & Yacu caspi & 3 \\
\hline 42 & Flacuortaceae & Caseria arborea & Maria & 9 \\
\hline 43 & Icacinaceae & Metteniusa tesmanniana & Palo de tacto & 4 \\
\hline 44 & Icacinaceae & Calatola aff. costaricensis & Tzintsala blanco & 1 \\
\hline 46 & Lauraceae & Ocotea leucoxylon & Canelo amarillo & 7 \\
\hline 47 & Lauraceae & Aniba coto & canelo bobo & 2 \\
\hline 48 & Lauraceae & Endlicheria griseo-sericea & Canelo Café & 2 \\
\hline 49 & Lauraceae & Rhodostemonodaphne napoensis & Canelo blanco & 2 \\
\hline 50 & Lauraceae & Nectandra membranaceae & Canelo de pantano & 1 \\
\hline 51 & Lauraceae & Ocotea sp. 1 & Canelo amarillo & 1 \\
\hline 52 & Lauraceae & Ocotea cernua & Canelo blanco & 1 \\
\hline 53 & Lauraceae & Pleurothyrium trianae & Canelo amarillo & 1 \\
\hline 54 & Lauraceae & Pleurothyrium glabrifolium & Canelo anís & 1 \\
\hline 55 & Lecythidaceae & Sweilera andina & Hualis yura & 1 \\
\hline 56 & Melastomataceae & Miconia affinis & Colca hoja mediana & 4 \\
\hline 57 & Melastomataceae & Miconia trinervia & & 12 \\
\hline 58 & Melastomataceae & Blakea rosea & Colca hoja fina & 3 \\
\hline 59 & Melastomataceae & Miconia pilgeriana & Colca & 6 \\
\hline 60 & Melastomataceae & Miconia splendens & Colca hoja ancha & 10 \\
\hline 61 & Melastomataceae & Miconia clathrantha & Colca & 3 \\
\hline 62 & Melastomataceae & Miconia pterocaulon & Colca hoja larga & 1 \\
\hline 63 & Melastomataceae & Miconia calvescens & Colca hoja ancha & 1 \\
\hline 64 & Melastomataceae & Miconia glanlulytyla & Colca hoja grande & 1 \\
\hline 65 & Meliaceae & Trichilia septentrionalis & Tucuta & 1 \\
\hline 66 & Meliaceae & Trichilia rubra & Tucuta & 3 \\
\hline 67 & Meliaceae & Cedrela odorata & Cedro & 3 \\
\hline 68 & Meliaceae & Guarea guidonia & Tucuta macho & 2 \\
\hline 69 & Meliaceae & Trichilia obovata & Tucuta & 4 \\
\hline 70 & Meliaceae & Cabralea canjerana & Cedrillo & 2 \\
\hline 71 & Meliaceae & Guarea kunthiana & Cedrillo & 7 \\
\hline 72 & Monimiaceae & Siparuna schimpffii & hoja de mal aire & 4 \\
\hline 73 & Monimiaceae & Mollinedia latifolia & Tzintzala & 4 \\
\hline 74 & Moraceae & Ficus trigona & Mata palo de pantano & 2 \\
\hline
\end{tabular}




\begin{tabular}{|c|c|c|c|c|}
\hline 75 & Moraceae & $\begin{array}{l}\text { Perebea guianensis ssp. } \\
\text { Pseudopeltata }\end{array}$ & Lengua de vaca & 1 \\
\hline 76 & Moraceae & Ficus insipida & Marrañun caspi & 1 \\
\hline 77 & Myristicaceae & Otoba parvifolia & Doncel & 2 \\
\hline 78 & Myristicaceae & Geissanthus longistamineus & Lengua de vaca & 1 \\
\hline 79 & Myrsinaceae & Ardisia guianensis & Roble blanco & 2 \\
\hline 80 & Myrtaceae & Myrcia aliena & Roble rojo & 1 \\
\hline 81 & Myrtaceae & Myrcia fallax & Roble blanco & 1 \\
\hline 82 & Myrtaceae & Eugenia multirimosa & Arrayan & 1 \\
\hline 83 & Nyctaginaceae & Neea spruceana & Roble blanco & 2 \\
\hline 84 & Nyctaginaceae & Neea divaricata & Tzintzala & 7 \\
\hline 85 & Piperaceae & Piper imperiale & Maria panga & 13 \\
\hline 86 & Poaceae & Guadua angustifolia & Guadua & 5 \\
\hline 87 & Proteaceae & Roupala montana & Negrocaspi & 1 \\
\hline 88 & Rubiaceae & Alibertia occidentalis & caimiton & 4 \\
\hline 89 & Rubiaceae & Joosia umbellifera & Intachic & 4 \\
\hline 90 & Rubiaceae & Faramea fragans & Tzembio & 6 \\
\hline 91 & Rubiaceae & Palicourea acanthaceae & Flor amarillo & 19 \\
\hline 92 & Rubiaceae & Faramea uniflora & Arrayan & 3 \\
\hline 93 & Rubiaceae & Coussarea ecuadorensis & Pepa de algodón & 13 \\
\hline 94 & Rubiaceae & Psychotria cuatrecasii & Flor amarillo & 4 \\
\hline 95 & Rubiaceae & Posoqueria latifolia & Desconocido Esp N & 1 \\
\hline 96 & Rubiaceae & Palicourea hospitalis & Flor amarillo & 20 \\
\hline 97 & Rubiaceae & Faramea glandulosa & Tzembio & 2 \\
\hline 98 & Rubiaceae & Alibertia edulis & Caimitón & 1 \\
\hline 99 & Rubiaceae & Psychotria ceronii & Caimitón & 1 \\
\hline 100 & Rubiaceae & Psychotria caerulea & Quilo & 6 \\
\hline 101 & Rutaceae & Zanthoxylum formiciferum & Tachuelo & 8 \\
\hline 102 & Rutaceae & Zanthoxylum riedelianum & Tachuelo & 12 \\
\hline 103 & Solanaceae & Brunfelsia grandiflora & Chiri caspi & 1 \\
\hline 104 & Staphylaceae & Turpinia occidentalis & Cuero de sapo & 1 \\
\hline \multirow[t]{2}{*}{105} & Violaceae & Gloeospermum equatoriense & Shulla yura & 1 \\
\hline & & & & 510 \\
\hline
\end{tabular}

Fuente: Investigación directa. 
Cuadro 2. Especies registradas en Bosque Secundario

\begin{tabular}{|c|c|c|c|c|}
\hline COD. & FAMILIA & NOMBRE CIENTÍFICO & ESPECIE & $\mathbf{F}$ \\
\hline 1 & Actinidaceae & Saurauia aequatoriensis & Moquillo & 58 \\
\hline 2 & Anacardiaceae & Tapirira aff. myriantha & Cedrillo & 1 \\
\hline 3 & Annonaceae & Annona duckei & Anona del monte & 1 \\
\hline 4 & Annonaceae & Guatteria sp. 1 & Sapán & 8 \\
\hline 5 & Arecaceae & Geonoma brongniartii & Chontilla blanca & 3 \\
\hline 6 & Arecaceae & Wettinia maynensis & Kilo & 7 \\
\hline 7 & Arecaceae & Prestoea schultzeana & chontillo amarillo & 1 \\
\hline 8 & Arecaceae & Socratea exorrhiza & Patona & 17 \\
\hline 9 & Asteraceae & Piptocoma discolor & Pigue & 34 \\
\hline 10 & Bombacaceae & Matisia soegengii & Sapote & 4 \\
\hline 11 & Brunelliaceae & Brunellia stenoptera & Cedrillo & 3 \\
\hline 12 & Burseraceae & Crepidospermun rhoifolium & Sapote blanco & 1 \\
\hline 13 & Caesalpinaceae & Senna bacillaris & Senna & 2 \\
\hline 14 & Caprifoliaceae & Viburnum toronis & Yacu caspi blanco & 48 \\
\hline 15 & Caprifoliaceae & Viburnum ayavacense & Yaku caspi blanco & 5 \\
\hline 16 & Cecropiaceae & Cecropia ficifolia & Guarumo & 13 \\
\hline 17 & Cecropiaceae & Cecropia angustifolia & Guarumo & 22 \\
\hline 18 & Cecropiaceae & Pouruma cecrofilia & Uva del monte & 1 \\
\hline 19 & Cecropiaceae & Cecropia sp. & Guarumo & 4 \\
\hline 20 & Clusiaceae & Vismia lateriflora & Achotillo & 16 \\
\hline 21 & Clusiaceae & Vismia pozuzoensis & Achotillo macho & 11 \\
\hline 22 & Costaceae & Costus laevis & Caña agria & 3 \\
\hline 23 & Cyathaceae & Cyathea sp. & Helecho & 1 \\
\hline 24 & Cyatheaceae & Cyathea bipinnatifida & Helecho arborecente & 1 \\
\hline 25 & Elaeocarpaceae & Sloanea guianensis & Palo de achote & 3 \\
\hline 26 & Euphorbiaceae & Sapium peruvianum & Caucho & 2 \\
\hline 27 & Euphorbiaceae & Tetrorchidium macrophyllum & Parecido al motilon & 1 \\
\hline 28 & Euphorbiaceae & Alchornea latifolia & Ponce & 26 \\
\hline 29 & Euphorbiaceae & Hyeronima oblonga & Motilon & 18 \\
\hline 30 & Euphorbiaceae & $\begin{array}{l}\text { Hyeronima scabrida (Tul.) Müll. } \\
\text { Arg. }\end{array}$ & Motión & 2 \\
\hline 31 & Euphorbiaceae & Croton tessmannii & Agujero & 1 \\
\hline 32 & Euphorbiaceae & Croton lechleri & Drago & 6 \\
\hline 33 & Flacuortaceae & Caseria arborea & Maria & 8 \\
\hline 34 & Lacistemataceae & Lozania klugii & Yacu caspi colorado & 64 \\
\hline 35 & Lauraceae & Pleurothyrium aff. trianae & Canelo bobo & 6 \\
\hline
\end{tabular}




\begin{tabular}{|c|c|c|c|c|}
\hline 36 & Lauraceae & Nectandra coeloclada & Canelo Blanco & 4 \\
\hline 37 & Lauraceae & Ocotea leucoxylon & Canelo Amarrillo & 2 \\
\hline 38 & Lauraceae & Ocotea cernua & Canelo alcanfor & 1 \\
\hline 39 & Lauraceae & Ocotea longifolia & Canelo bobo & 1 \\
\hline 40 & Lauraceae & Rhodostemonodaphne napoensis & Canelo k k & 5 \\
\hline 41 & Lauraceae & Endlicheria griseo-sericea & Canelo bobo & 1 \\
\hline 42 & Melastomataceae & Miconia splendens & Colca Amarrilla & 46 \\
\hline 43 & Melastomataceae & Miconia quadripora & Colca & 4 \\
\hline 44 & Melastomataceae & Miconia cercophora & Colca & 1 \\
\hline 45 & Melastomataceae & Miconia calvescens & Colca hoja apiculada & 3 \\
\hline 46 & Melastomataceae & Miconia affinis & Colca hoja mediana & 4 \\
\hline 47 & Melastomataceae & Miconia glaucesans & Colca hoja mediana & 1 \\
\hline 48 & Melastomataceae & Miconia smaragdina & Colca hoja & 18 \\
\hline 49 & Melastomataceae & Miconia aff. triplenervis & colca hoja mediana & 1 \\
\hline 50 & Melastomataceae & Miconia lugonis & Colca hoja fina & 5 \\
\hline 51 & Melastomataceae & Miconia trinervia & Colca bp & 6 \\
\hline 52 & Melastomataceae & Miconia pilgeriana & Colca hoja mediana & 29 \\
\hline 53 & Meliaceae & Guarea humaitensis & Tucuta & 1 \\
\hline 54 & Meliaceae & Trichilia rubra & Tucuta & 1 \\
\hline 55 & Meliaceae & Trichilia septentrionalis & Tucuta & 1 \\
\hline 56 & Meliaceae & Cabralea canjerana & Cedrillo & 4 \\
\hline 57 & Mimosaceae & Inga multinervis & Guaba del río & 3 \\
\hline 58 & Mimosaceae & Inga acreana & Guaba del monte & 1 \\
\hline 59 & Mimosaceae & Inga aff. tessmannii & Guaba de monte & 4 \\
\hline 60 & Mimosaceae & Inga oerstediana & Guaba bejuca de monte & 7 \\
\hline 61 & Mimosaceae & Calliandra trinervia & Yutzo & 5 \\
\hline 62 & Mimosaceae & Inga tessmanni & Guaba del monte & 5 \\
\hline 63 & Mimosaceae & Zygia coccinea & Guabilla & 4 \\
\hline 64 & Mimosaceae & Inga velutina & Guaba de río & 9 \\
\hline 65 & Monimiaceae & Siparuna schimpffii & Suna panga & 7 \\
\hline 66 & Monimiaceae & Mollinedia latifolia & Sinzala blanco & 2 \\
\hline 67 & Moraceae & $\begin{array}{l}\text { Perebea guianensis ssp. } \\
\text { Pseudopeltata }\end{array}$ & Lengua de vaca & 1 \\
\hline 68 & Myrsinaceae & Myrsine coriaceae & Alamo & 1 \\
\hline 69 & Myrsinaceae & Ardisia panurensis & Motilón rojo & 1 \\
\hline 70 & Myrtaceae & Eugenia patrisii & Arrayan blanco & 1 \\
\hline 71 & Myrtaceae & Eugenia florida & Madera blanca & 1 \\
\hline 72 & Myrtaceae & Eugenia muricata & Guayabillo & 2 \\
\hline
\end{tabular}




\begin{tabular}{|c|c|c|c|c|}
\hline 73 & Myrtaceae & Eugenia multirimosa & Roble & 2 \\
\hline 74 & Myrtaceae & Myrcia aliena & Roble blanco & 1 \\
\hline 75 & Myrtaceae & Myrcia fallax & Roble rojo & 1 \\
\hline 76 & Piperaceae & Piper aduncum & Matico silvestre & 3 \\
\hline 77 & Piperaceae & Piper imperiale & Maria panga & 7 \\
\hline 78 & Proteaceae & Roupala montana & Negro caspi & 2 \\
\hline 79 & Rubiaceae & Palicourea gomezii & Moradilla & 27 \\
\hline 80 & Rubiaceae & Palicourea guianensis & Moradilla & 23 \\
\hline 81 & Rubiaceae & Policaurea acanthaceae & Flor amarilla & 7 \\
\hline 82 & Rubiaceae & Psychotria caerulea & Arbusto manera cruz & 2 \\
\hline 83 & Rubiaceae & Alibertia occidentalis & Caimiton & 1 \\
\hline 84 & Rubiaceae & Faramea salicifolia & Arrayan colorado & 2 \\
\hline 85 & Rubiaceae & Psychotria ceronii & Calmitón & 1 \\
\hline 86 & Rubiaceae & Psychotria cuatrecasii & Flor amarilla & 8 \\
\hline 87 & Rubiaceae & Coussarea ecuadorensis & Palo blanco mindal & 2 \\
\hline 88 & Rubiaceae & Isertia laevis & Hoja de espuma & 1 \\
\hline 89 & Rubiaceae & Joosia umbellifera & Intachic & 3 \\
\hline 90 & Rubiaceae & Palicourea condorica & Flor amarilla & 1 \\
\hline 91 & Rutaceae & Esenbeckia amazonica & Tsicta & 1 \\
\hline 92 & Rutaceae & Zanthoxylum formiciferum & Tachuelo & 4 \\
\hline 93 & Rutaceae & Zanthoxyum kellermanii & Tachuelo & 1 \\
\hline 94 & Solanaceae & Solanum amisophyllum & Ajo blanco & 1 \\
\hline 95 & Solanaceae & Solanum grandiflorum & Naranjilla silvestre & 1 \\
\hline 96 & Staphylaceae & Turpinia occidentalis & Cuero de sapo & 2 \\
\hline 97 & Sterculiaceae & Herrania nycterodendron & Cacao de monte & 1 \\
\hline 98 & Urticaceae & Urera baccifera & Ortiga & 7 \\
\hline 99 & Vochysiaceae & Vochysia aff. bracelinae & Tamburo & 1 \\
\hline \multirow[t]{2}{*}{100} & Vocysiaceae & Vochysia braceliniae & Tamburo blanco & 3 \\
\hline & & & & 711 \\
\hline
\end{tabular}


